\title{
Present performance of the dark-speckle coronagraph ${ }^{\star}$
}

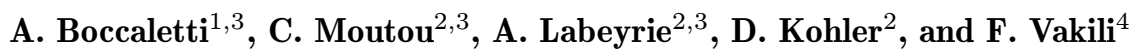 \\ 1 DESPA, Observatoire de Paris-Meudon, 5 place J. Janssen, F-92195 Meudon, France \\ e-mail: Boccalet@despa.obspm.fr \\ 2 Observatoire de Haute-Provence, F-04870 St-Michel l'Observatoire, France \\ e-mail: Moutou@obs-hp.fr, Labeyrie@obs-hp.fr \\ ${ }^{3}$ Collège de France, 11 place M. Berthelot F-75321 Paris, France \\ 4 Observatoire de la Côte d'Azur, Département Fresnel, GI2T, F-06460 Saint Vallier de Thiey, France \\ e-mail: Vakili@obs-azur.fr
}

Received March 19; accepted June 17, 1998

\begin{abstract}
We present the recent developments of the dark speckle coronagraph and results obtained on two close binary stars. The central star is occulted by a Lyot coronagraphic system and the residual speckle pattern is detected on a photon-counting camera. Achromaticity is achieved across a wide spectral band, using a Wynne corrector. We observed the relatively faint companions of $\delta$ Per and $\eta$ Psc and give an independent estimate of their position and magnitude difference.
\end{abstract}

Key words: planetary systems - stars: $\delta$ Per, $\eta$ Psc, $\eta$ And - instrumentation: interferometers; miscellaneous

\section{Introduction}

Even after the conclusion of the controversy on the 51 Pegasi planet (Gray 1997; Gray 1998; Mayor \& Queloz 1995), the lack of real images remains a major difficulty in interpreting indirect detections of exoplanets. Direct imaging is classically hampered by the huge intensity ratio between a solar-type star and its planets. It requires spatial resolution beyond the atmopsheric limit, together with efficient nulling of the star light (Angel 1994). Highperformance adaptive optics are being developed to improve the instrument performance (Angel 1994; Gezari et al. 1997b). New approaches to stellar coronagraphy have also been proposed recently: the phase mask system (Roddier \& Roddier 1997) and the achromatic interferometric coronagraph AIC (Gay \& Rabbia 1996). Both are expected to achieve a higher sensitivity than the Lyot

Send offprint requests to: A. Boccaletti

* Based on data collected at the Observatoire de HauteProvence. coronagraph, and should allow the search for the companion closer to the central star (a fraction of the Airy disk) than the Lyot system (a few Airy disks).

Our instrument is a visible Lyot coronagraph which uses the dark-speckle detection (Labeyrie 1995) for exoplanet imaging. It stops the star's Airy peak and rings restored by an adaptive optics system. The method exploits the occurrence of dark speckles (destructive interferences in the focal plane) to clean the halo of scattered light. The statistical analysis of this occurrence shows positions where faint planet images prevent full darkening of the star's speckles. The depletion of zero-photon count in these regions thus indicates the presence of faint companions. By processing a large number of short exposures, faint companions can emerge above the residual noise.

A refined theoretical analysis of dark-speckle imaging is given in Boccaletti et al. (1998), together with numerical and laboratory simulations. Simulations have shown, for the case of the Hubble Space Telescope, that imaging an exoplanet with $10^{-9}$ intensity ratio is possible with a darkspeckle coronagraph in a few hours (Boccaletti 1998).

The first observation, in june 1996, at the Observatoire de Haute-Provence of the binary star HD $144217(\Delta m=$ 4.8) has confirmed the simulations (Boccaletti et al. 1998).

Here, we describe recent improvements of our instrument (Sect. 2) and some results obtained during a tennight observing run in october 1997 at the Observatoire de Haute-Provence (Sect. 3). To test the dark-speckle method and gain experience in high sensitivity coronagraphic imaging, we observed Hipparcos binaries having large magnitude differences. These instrumental developments also prepare the next generation of dark-speckle coronagraphs, to be used on large ground-based or space telescopes (Gezari et al. 1997b) and interferometric arrays (Labeyrie 1998). 


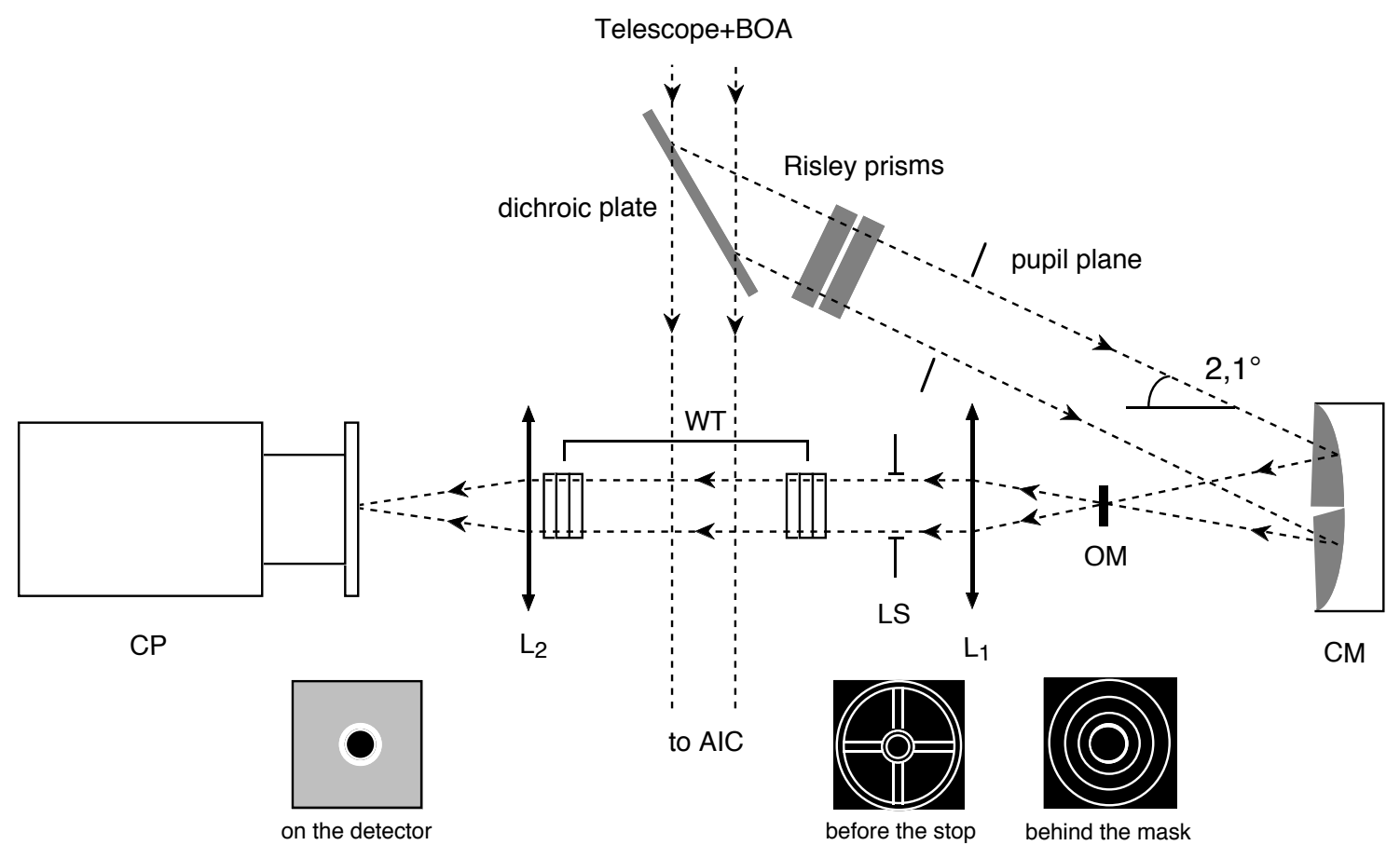

Fig. 1. Dark speckle camera used at the Haute-Provence $1.52 \mathrm{~m}$ telescope (not a scale drawing). The dichroic beam-splitter selects the wavelength range $650-850 \mathrm{~nm}$ in reflection. The collimated beam received from the telescope with BOA adaptive optics crosses a pair of Risley prisms for atmospheric dispersion compensation and is focussed by concave mirror CM onto the Lyot occulting mask OM. The masked field is re-imaged onto photon-counting camera CP through the pair of Wynne corrector triplets WT which compensate the first-order lateral chromatism of diffraction patterns. A Lyot stop LS removes stellar diffracted light at the edge of the relayed pupil, according to the classical Lyot coronagraph principle

\section{Instrument layout}

\subsection{General view}

The dark-speckle coronagraph (DSC) combines features of adaptive optics, Lyot coronagraphy and speckle interferometry. Figure 1 shows the arrangement utilized at the Coudé focus of the $152 \mathrm{~cm}$ telescope at Observatoire de Haute-Provence. The stellar beam from the telescope enters the adaptive optics bench BOA, of the ONERA (Office National d'Études et de Recherches Aérospatiales). It has 88 actuators and responds fast enough for seeing correction in the visible range (Conan et al. 1998). Downstream from BOA, the afocal beam reaches a dichroic beamsplitter which transmits the IR ( $K$ band) light towards another instrument used simultaneously, the achromatic interfero-coronagraph (Gay \& Rabbia 1996), while visible light $(650 \mathrm{~nm}<\lambda<850 \mathrm{~nm})$ is reflected to the DSC.

The Strehl ratio reached $10 \%$ to $30 \%$ depending on seeing conditions. Although the adaptive optics gain is higher in the IR range, the advantage of working with visible light results from the use of a photon-counting camera. Its low dark count allows short exposures with negligible added noise, as required for speckle observations.

The centering of the star's image on the Lyot occulting mask is critical, and should be part of the adaptive optics loop. This is achievable by collecting light reflected from the mask, but the drift had to be corrected manually at this stage.

\subsection{Optical bench}

\subsubsection{Coronagraph}

The diffraction rings are removed by a Lyot coronagraph (Lyot 1930, Fig. 1). The occulting masks in the f/122 beam are inter-changeable, with sizes correponding to $0.22^{\prime \prime}, 0.45^{\prime \prime}$ and $0.67^{\prime \prime}$. They have been vaccum evaporated through pinholes onto a $1.2 \mathrm{~mm}$ thick plate of antireflection coated glass. The apodizing masks located at the relayed pupil are pinholes carried by a $x-y$ translation stage. No attempt was made to mask the aperture spider and central obscuration although this should decrease the scattered light significantly. 


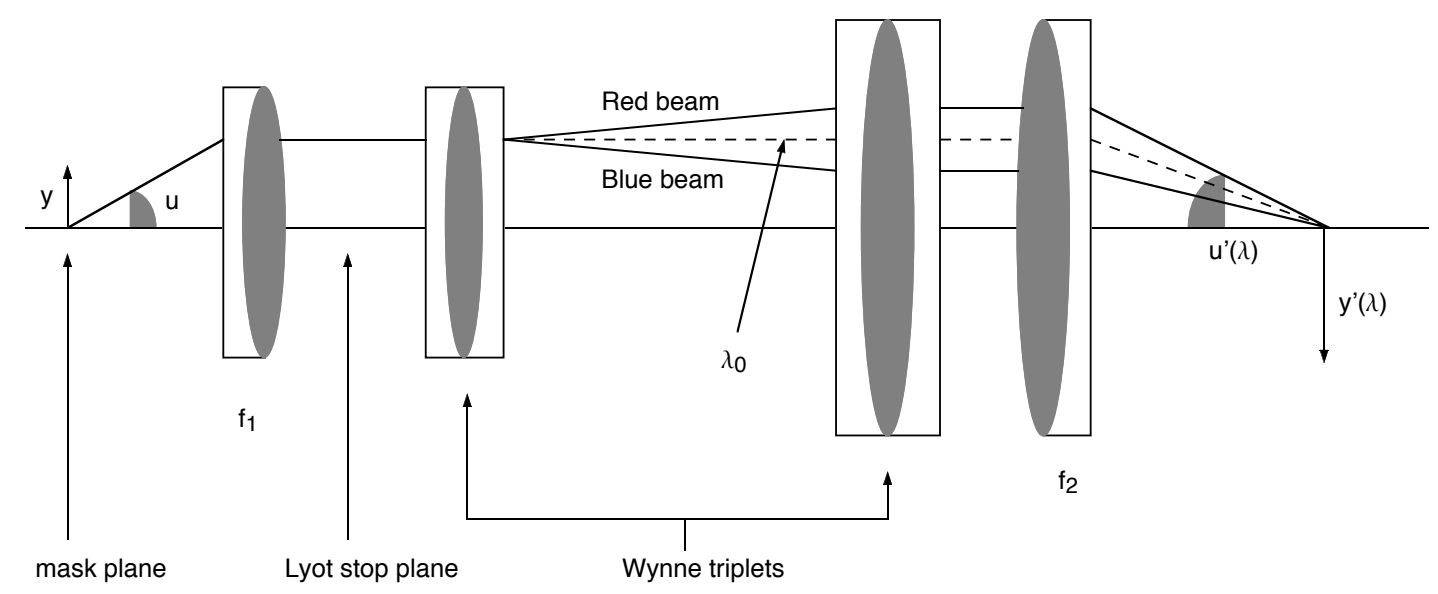

Fig. 2. Wynne corrector for making the speckle pattern wavelength-invariant. A pair of triplets, afocal in yellow light, shrinks the blue pupil while enlarging the red pupil. The exit angle, $u^{\prime}$, becomes approximately proportional to wavelength, thus ensuring a nearly wavelength-invariant diffraction pattern

\subsubsection{Atmospheric dispersion}

The atmospheric dispersion corrector, located near the first pupil image, uses a pair of normal-field Risley prisms, which are rotated. The glasses, Schott PK51A and Corning B29-52, correct a wide spectral bandpass, from $380 \mathrm{~nm}$ up to $950 \mathrm{~nm}$. The residual chromatism in the $600-850 \mathrm{~nm}$ band is on average $1 / 4$ of the Airy peak diameter, corresponding to $0.03^{\prime \prime}$ at $750 \mathrm{~nm}$. With the $1.5 \mathrm{~m}$ telescope and the $6 \mathrm{~mm}$ pupil, the prisms correct up to $60^{\circ}$ from zenith. We adjusted visually the prism settings although pre-calculated settings would be preferable, especially for faint stars.

\subsubsection{Wynne correctors}

For conventional speckle interferometry the detected bandwidth can be increased by transforming the partially proportional wavelength dependence of the speckle scale. Here, the proportionality is more accurate since the residual phase on the wave is made small by the adaptive optics. The photon rate being critical in ground-based darkspeckle imaging, there is much to gain in increasing the usable spectral bandwidth.

One of us (DK) has re-calculated the corrector solution obtained by Wynne (1979) (Fig. 2). The pair of null triplets shrinks the blue pupil, while enlarging the red pupil, so that both Airy patterns, or speckle patterns, are of nearly identical size. With respect to the original Wynne design, we have suppressed the power in the second triplet, in order to keep more flexibility in the final magnification and we have replaced the SF 8 glass by SFL5 for optimal correction in the red range $(600-850 \mathrm{~nm})$.

- At $\lambda_{0}=635 \mathrm{~nm}$, the glasses have the same refractive index and the two triplets behave as two plane-parallel plates.
- System performance is limited by the non-linearity of the glass dispersion. In the range $600-850 \mathrm{~nm}$, the final radial chromatism, including diffraction, is lower than $1.7 \%$ of the axial distance (Fig. 6).

- The corrected field is quite small owing to the strong curvatures which limit the diameter of the exit triplet.

- The star image must be kept on axis, and any companion has its Airy peak dispersed, although not very much with the small relative spectral bandwidth utilized. Field spectrocopy techniques (Bacon et al. 1995) or wavelength sensitive detectors such as the Superconductive Tunnel Junction camera (Peacok et al. 1998) could solve this residual problem.

- Although it would be nice to insert the occulting mask in the achromatised image, the following pupil would become chromatic, which would complicate the apodization masking.

The analytical derivation is given in Appendix.

\subsubsection{The photon-counting camera}

Finally $L_{2}$ focuses the beam onto the camera with an $8 \times$ magnification, bringing the aperture to $f / 976$.

The detector is a cooled CP20 photon-counting camera (Vakili 1990) having a first-generation electrostatic intensifier coupled to a second-generation microchannel intensifier and a fiber optics taper, feeding a $384 \times 288$ CCD. The resulting amplification is about $10^{5}$. Such a device allows single photon detection and a very low dark count, less than 10 photons per $20 \mathrm{~ms}$ exposure for $50 \mu \mathrm{m}$ pixels at $-20^{\circ} \mathrm{C}$ (about 0.0045 photons/s/pixel). A low dark noise is necessary to detect accurately any "filling" of the dark speckles, which could reveal the presence of a faint companion. The drawbacks of such photon-counting cameras are their low quantum efficiency $(<10 \%$ at $700 \mathrm{~nm})$ and their low saturation level of $50000 \mathrm{ph} / \mathrm{s}$, limited by the acquisition system. 


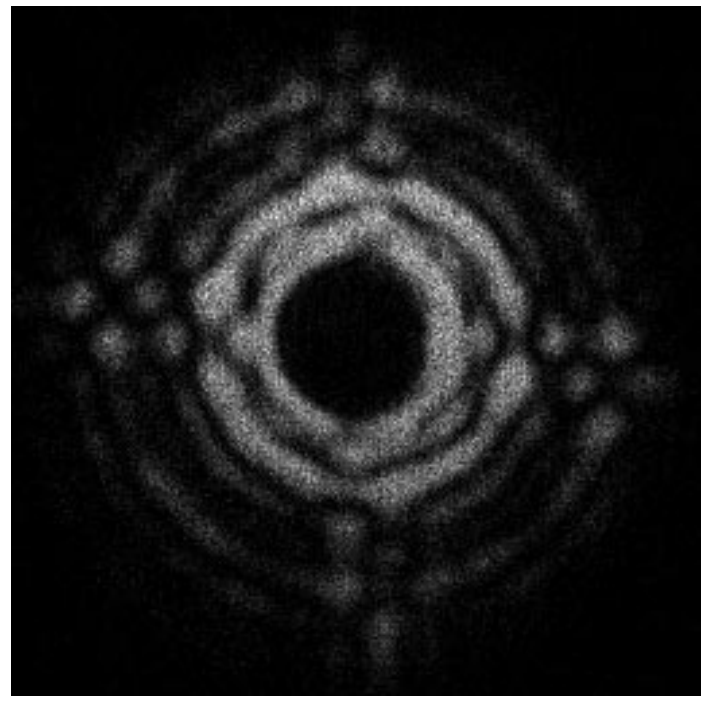

Fig. 3.a) Reference image obtained with a white light source inside the BOA adaptive optics system, using the $0.45^{\prime \prime}$ mask and without the Lyot stop. The Airy rings remain visible. Diffraction spikes from the spider arms can be seen as dotted lines, influenced by the ring structure. In such conditions a planetary companion would be invisible. In spite of the broad spectral bandwidth $(\Delta \lambda=650-850 \mathrm{~nm})$ at $\lambda_{0}=635 \mathrm{~nm}$, the outer rings retain good contrast, owing to the Wynne corrector

As mentioned above, the coronagraph forms a $f / 976$ focus on the detector in order to achieve the dense sampling required by the dark-speckle technique. For a central wavelength of $635 \mathrm{~nm}$, it represents 150 pixels/speckle area or 144 pixels/arcsecond. The field of view is limited to a diameter of 250 pixels (about $1.8^{\prime \prime}$ ) by the Wynne corrector. In addition to the Wynne corrector, spectral filters can be inserted in front of the photocathode to select different narrow bandwidths.

\subsection{Efficiency of the Lyot stop}

To calibrate the efficiency of the Lyot stop, we have acquired an internally-generated reference image using a single-mode fiber included in BOA. The Strehl ratio $(S R)$ of this reference source is about $80 \%$ and does not take into account the atmospheric turbulence or the static aberrations of the adaptive mirror. When the core of the Airy pattern is occulted by the mask, the edges of the pupil image become decorated with two bright fringes (Fig. 1). The complementary spatial filter in the pupil plane should suppress much of the diffracted light, except that caused by the wave bumpiness. The Lyot stop being a simple pinhole of $400 \mu \mathrm{m}$ instead of a telescope pupil image, some Airy-like rings remain visible in the final image (Fig. 3b). Moreover, the spider spikes combined with the residual rings produce symmetrical side-lobes, especially bright within the 2 first rings. These artifacts remain on the compensated images despite the smoothing introduced by the atmospheric turbulence.

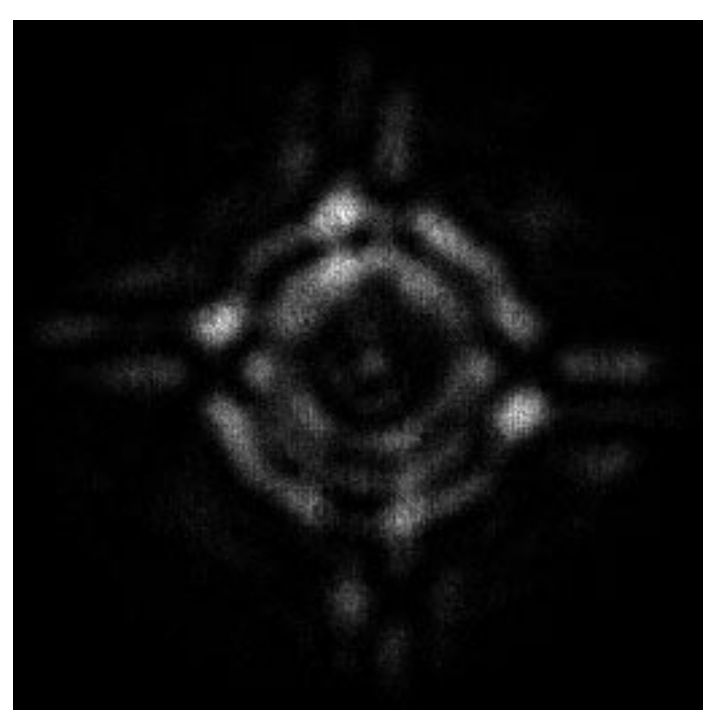

Fig. 3.b) The same reference frame obtained with the mask and the $400 \mu \mathrm{m}$ Lyot stop. Here, the Airy pattern is markedly attenuated and the gain in sensitivity is about 1.7 magnitudes. Nevertheless, due to the inadapted Lyot stop, some bright features remain, like the rings around the mask and the four symmetrical speckles which could bring out wrong detections. These static defects can be partially removed with frame subtraction

One can compute the rejection rate of the coronagraph as defined in Malbet 1996:

$R=\frac{I_{\mathrm{w} / \mathrm{o}}}{I_{\mathrm{w}}}$

where $I_{\mathrm{w} / \mathrm{o}}$ is the total intensity of the reference beam without coronagraph and $I_{\mathrm{w}}$ is its intensity with the coronagraph. To characterize the efficiency of the Lyot stop, one defines $R_{\mathrm{w}}$ the rejection rate with the Lyot stop and $R_{\mathrm{w} / \mathrm{o}}$ without it. We can then estimate the gain in magnitude introduced by the Lyot stop with the following relation (Malbet 1996):

$\Delta m=2.52 \log \left(2 R_{\mathrm{w}} / R_{\mathrm{w} / \mathrm{o}}\right)$.

A gain of 1.7 magnitude has been measured for the $0.45^{\prime \prime}$ mask and the $400 \mu \mathrm{m}$ Lyot stop. As this value is averaged over the entire field, it is therefore underestimated far from the axis and overestimated near the mask. An optimized Lyot stop, including secondary mirror and spider arms, should improve the gain by another 1.3 magnitude (Malbet 1996).

\section{Results for 2 binary stars}

\subsection{Data reduction}

A malfunction in the camera control computer caused the loss of $80 \%$ to $90 \%$ of the short exposures. As a consequence, the speckle noise remains 3 times more intense than expected and dominates the photon noise. 


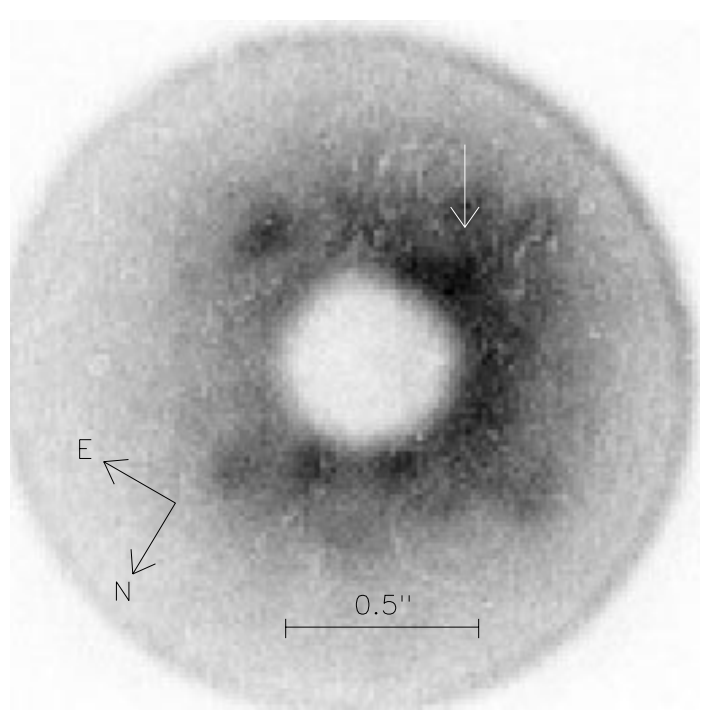

Fig. 4.a) Negative cleaned image of the binary star $\delta$ Per, obtained in $\mathrm{H} \alpha(\lambda=653.6 \mathrm{~nm}, \Delta \lambda=10 \mathrm{~nm})$ with the $0.45^{\prime \prime}$ mask. The intensity of the image represents the number of zero photon collected with 9503 short exposures of $20 \mathrm{~ms}$. The hypothetic companion (arrow) is visible at the edge of the mask $\left(\mathrm{PA}=202^{\circ}, \rho=0.293^{\prime \prime}\right)$ as a "hole" in the cleaned map, amidst the fixed speckles generated by the spider structure. At this location, the zero photon-count is lower than elsewhere

The dark-speckle algorithm is described in Labeyrie (1995) and Boccaletti et al. (1998). The data reduction software provides two images. The first is equivalent to a long exposure, computed by co-adding all the short exposures containing the position of the photon-events. The second image, called the "cleaned map", displays the result of the dark-speckle algorithm. The number of zerophoton events occurring in binned pixels containing $2 \times 2$ camera pixels, is cumulated over successive frames. The cleaned map, thus obtained in negative form, can be inverted with a suitable gamma exponent. In principle, the cleaned image is more sensitive to the faint structures than the conventional long exposure.

Photon-counting data are affected by the so-called photon-centroiding (Tiébaut 1994) resulting from the limited resolution of the electronics in space and time. The counting behaviour is expected to improve with forthcoming detectors. The signal to noise ratio (SNR) is measured in the cleaned map according to Eq. (6) from Labeyrie (1995). The magnitude difference is derived from the CP20 long exposure by comparing the flux of the star outside the mask, and the flux of the companion when the star is under the mask.

To assess the performance of the system, one can compute the expected maximum brightness ratio theoretically reachable (Eq. (7) Boccaletti et al. 1998). This value only depends on the sampling (150 pixels/speckle area) and on the adaptive optics (AO) gain which is the brightness ratio between the peak of the corrected image and the halo level. The average AO gain obtained in the present run is

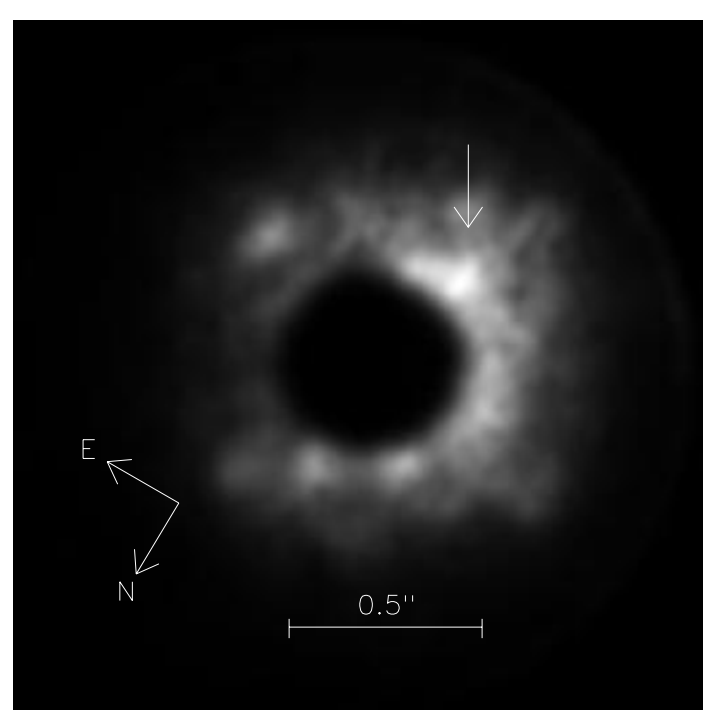

Fig. 4.b) The same image enhanced with an inverse square law, and smoothed to the speckle scale with a wavelet transform filter. The faint companion $(\Delta m=3.5)$ emerges above the speckle noise, although no reference star was available in this case to subtract the fixed speckles

about 15 at $0.5^{\prime \prime}$ from the star. The total integration time is assumed to be very large compared to the speckle lifetime. This leads to a 8.8 magnitude difference. However, as described in Sects. 3.2 and 3.3, this goal, although modest with respect to the $\Delta m=22$ goal attainable in principle with future adaptive optics, was not reached owing to practical problems. Among these was the loss of $90 \%$ of the observing data, due to a computer failure.

\section{2. $\delta$ Per}

The first target star observed is $\delta$ Per (HD22928, $m_{v}=$ $2.99, \mathrm{JD}=50725.646)$, a binary system previously observed by Hipparcos (ESA 1997). We used the 0.45" occulting mask and $400 \mu \mathrm{m}$ pupil stop. The turbulence conditions were characterized by $r_{0}=8 \mathrm{~cm}$ during observation. 9503 short exposures of $\delta$ Per have been acquired, corresponding to an observing time of $190 \mathrm{~s}$. We used an $\mathrm{H} \alpha$ filter $(653.6 \mathrm{~nm})$ of bandwidth $10 \mathrm{~nm}$. The faint companion appears at $\mathrm{PA}=(202 \pm 1)^{\circ}$ and $\rho=$ $(0.293 \pm 0.027)^{\prime \prime}$. The sampling on the camera is 144 pixels per arcsecond. The image (Figs. $4 \mathrm{a}$ and $4 \mathrm{~b}$ ) presents some fixed patterns, mainly produced by the spider arm diffraction which is visible as four symmetrical bright speckles. An AO gain of 6, azimuthally averaged, is obtained at $0.3^{\prime \prime}$ from the star. An SNR of 53 is measured on the speckle size $(15 \times 15$ pixels $)$, while the model predicted an SNR of 133 (Boccaletti et al. 1998). The discrepancy with theory can be an indirect effect of the computer failure mentioned above; speckle noise becomes dominant owing to the small number of exposures. The intensity of the companion was derived from the CP20 long exposure and 


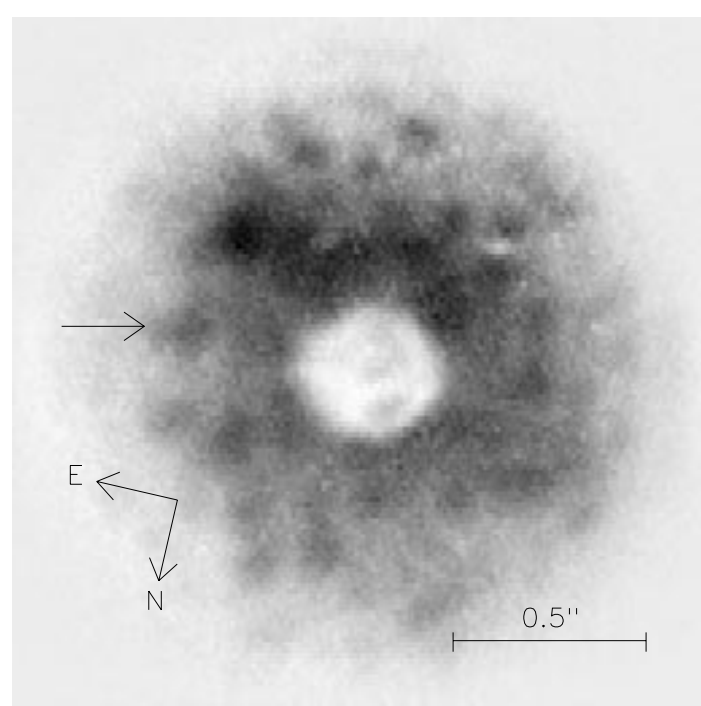

Fig. 5.a) Negative cleaned map of the binary star $\eta$ Psc, observed with the $0.45^{\prime \prime}$ mask and the Wynne corrector's spectral band $\left(\lambda_{0}=635 \mathrm{~nm}, \Delta \lambda=650-850 \mathrm{~nm}\right)$. Due to the strong turbulence $\left(r_{0}=6.2 \mathrm{~cm}\right)$ and the small number of exposures (9013), the faint companion (arrow) remains buried among the speckles. A flat field correction has been applied to the map

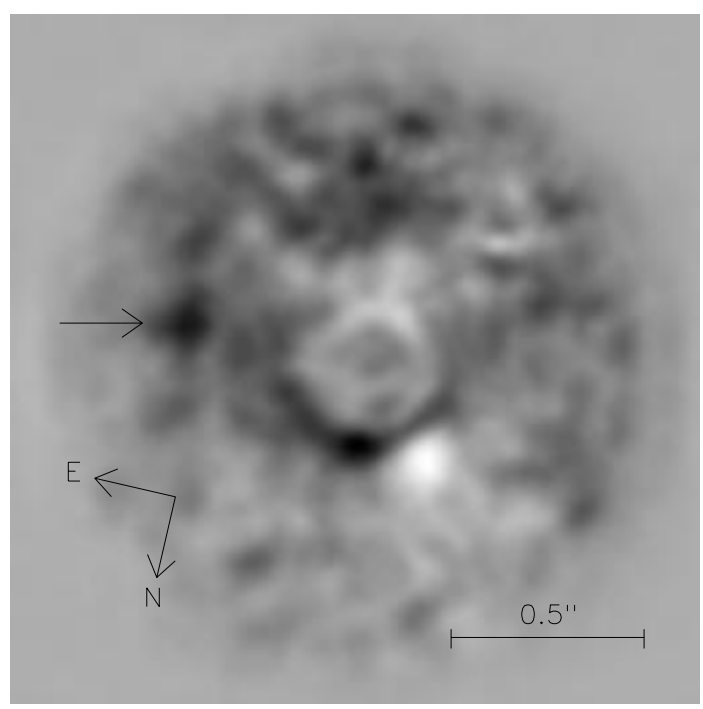

Fig. 5.c) Difference of $\eta$ Psc and $\eta$ And cleaned maps. The companion now appears as a dark feature (arrow). The image has been smoothed with a wavelet transform filter

corresponds to a $\Delta m$ of $3.48 \pm 0.20$. The second target star, $\eta$ Psc (HD 9270, $m_{v}=3.61$, JD $\left.=50730.507\right)$, was also selected from the Hipparcos binary star catalogue. Coronagraphic images of $\eta$ Psc were recorded without spectral filter, the bandpass being limited by the dichroic beam-splitter and the camera $(\Delta \lambda=650-850 \mathrm{~nm})$. These images illustrate the problem of the speckle noise in the search for faint companions. The Hipparcos data gives $\Delta m_{v}=3.14, \rho=0.33^{\prime \prime}$ and $\mathrm{PA}=221^{\circ}$, which is in good agreement with our results.

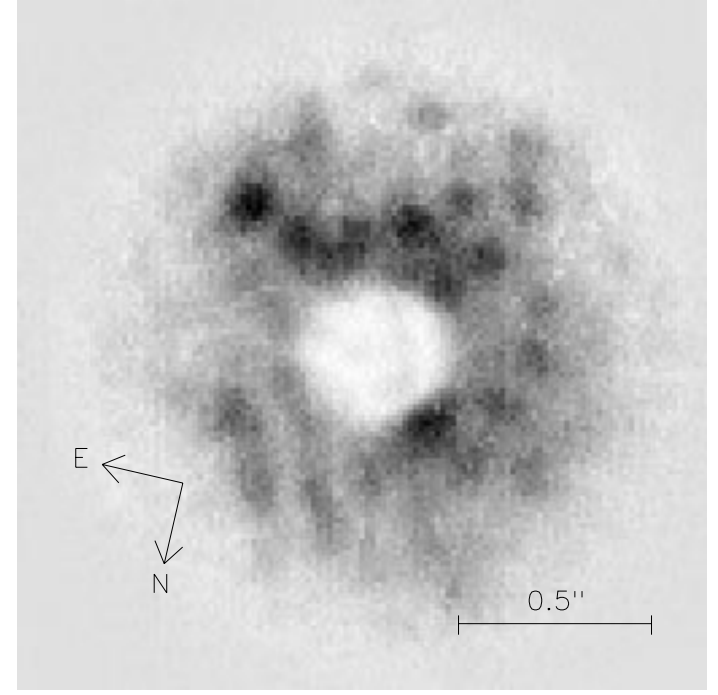

Fig. 5.b) Reference star ( $\eta$ And) observed in the same way just before $\eta$ Psc with $r_{0}=8 \mathrm{~cm}, 3645$ short exposures. The suspected "companion" peak of Fig. 5a is here absent, although the static residual speckles are similar. The scattered halo level is significantly lower than in Fig. $4 \mathrm{a}$

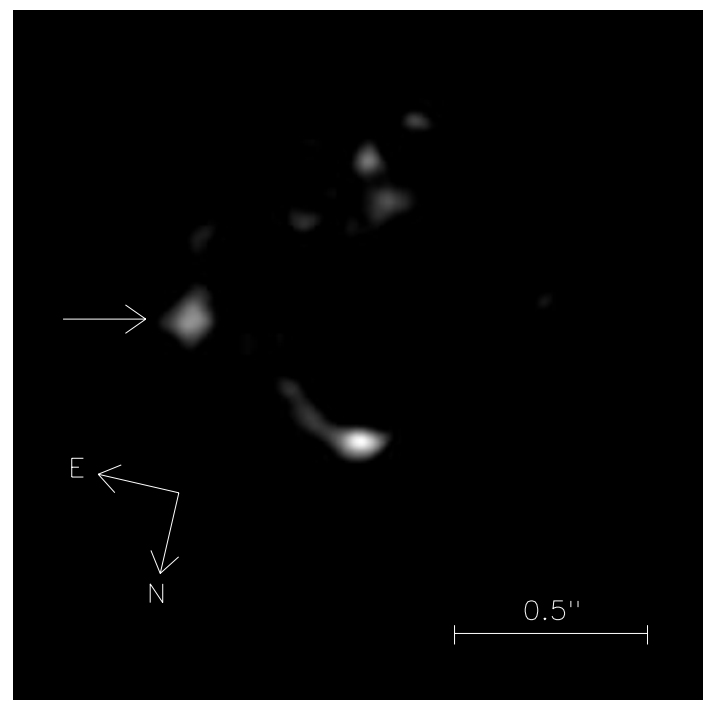

Fig. 5. d) Positive cleaned image obtained with an inverse square law, and a threshold at $3 \sigma$ level

\section{3. $\eta P s c$}

The second target star, $\eta$ Psc (HD 9270, $m_{v}=3.61$, $\mathrm{JD}=50730.507)$, was also selected from the Hipparcos binary star catalogue. Coronagraphic images of $\eta$ Psc were recorded without spectral filter, the bandpass being limited by the dichroic beam-splitter and the camera $(\Delta \lambda=650-850 \mathrm{~nm})$. These images illustrate the problem of the speckle noise in the search for faint companions. Indeed, for the same reason as above the number of short exposures is poor (9013). Altough the companion emerges easily above the photon noise, the 
residual speckle noise inhibits its detection on the raw data (Fig. 5a). However, a nearby reference star $\left(\eta\right.$ And, $m_{v}=$ 4.42), has been observed immediately afterwards for map subtraction (Fig. 5b). The efficiency of the subtraction is limited for several reasons:

(i) The conditions of turbulence, and thus the correction applied by the AO system, are different for both stars $\left(r_{0}=8 \mathrm{~cm}\right.$ for $\eta$ And, and $r_{0}=6.2 \mathrm{~cm}$ for $\eta \mathrm{Psc}$ ).

(ii) The coronagraphic images are not invariant to translation (Malbet 1996), and in fact highly sensitive to tracking drift. Any centering difference between both sequences causes spurious speckle noise in the subtracted image.

(iii) A $76 \mathrm{~Hz}$ vibration of the telescope drive motor, seen by the Shack-Hartmann wave sensor, is left uncorrected by the adaptive system.

To overcome these problems, both star and reference have been corrected from Flat-Field and smoothed with wavelet transform. Then, to account for the lower speckle contrast of $\eta$ Psc, presumably caused by unequal seeing lifetime during both observations, the reference star was convolved with a gaussian shape of 4 pixels width. Finally, $\eta$ And was shifted to overlap $\eta$ Psc in the field, and scaled in intensity (Fig. 5c). After subtraction of the two cleaned negative images, the remaining bright and dark speckles belong respectively to the reference star ( $\eta$ And) and the target star ( $\eta$ Psc).

The measurement of the SNR, on the cleaned map, is made difficult by the crowded field. Although the companion remains undetected on the initial image (Fig. 5a), in terms of speckle noise, the comparison of both images eliminates all features common to the target (Fig. 5a) and the reference (Fig. 5b), so that the only residual speckle indicates the companion position $\left(\rho=(0.507 \pm 0.01)^{\prime \prime}\right.$, $\left.\mathrm{PA}=(94 \pm 1)^{\circ}\right)$. This process leads to an SNR of 137 , instead of 250 as predicted by the model. In this case, the SNR is naturally decreased by the companion's smearing, induced by the Wynne correctors.

The long exposure allows to derive a $\Delta m=4.15 \pm 0.20$ in the $R$ band. The Hipparcos $V$-band data gives $\Delta m_{v}=$ 3.7, $\rho=0.64^{\prime \prime}$ and $\mathrm{PA}=47^{\circ}$. A difference of spectral types for both components can explain the apparent discrepancy. We must also notice a discrepancy in the companion position. As the orbital solution is unknown, further observing runs will be needed to confirm the detection of the companion.

\section{Further developments}

The following improvements of the instrument are foreseen:

- Improved adaptive stabilisation of the occulter centering.
Table 1. Summary of our results compared to the HIC data

\begin{tabular}{|l|c|c|}
\hline Star name & $\delta$ Per & $\eta$ Psc \\
\hline$m_{\mathrm{r}}$ & 2.99 & 3.61 \\
\hline$\Delta \lambda(\mathrm{nm})$ & 10 & 200 \\
\hline $\mathrm{PA}\left(^{\circ}\right)$ HIC/this paper & $221 / 202$ & $47 / 94$ \\
\hline$\Delta m$ HIC/this paper & $3.14 / 3.48$ & $3.64 / 4.15$ \\
\hline$\rho\left(^{\prime \prime}\right)$ HIC/this paper & $0.33 / 0.29$ & $0.64 / 0.51$ \\
\hline reference & no & $\eta$ And \\
\hline
\end{tabular}

- Lyot stop: as mentioned in Sect. 2.3, the use of a pinhole-shaped diaphragm introduces residual optical defects, such as static bright speckles at the crossing of a spider arm and the second Airy ring. For an optimization of the apodizing device, we will use in the future a diaphragm matching the telescope pupil shape. - The Risley prisms adjustement needs to be automated.

- Optimized detectors: regarding the photon-counting detector, AsGa photocathodes now available extend the photon-counting range to $900 \mathrm{~nm}$. They will be used on future photon-counting cameras. With their clean detection of photon-events, electron bombarded CCDs appear of interest, in spite of the rather slow readout currently available. Non intensified photoncounting CCDs are also announced.

- Speckle noise: In the cleaned image generated by the dark speckle algorithm, and also in a conventional long-exposure a fixed pattern of speckles tends to remain visible in the smoothed halo.

Long integrations, whether in the dark-speckle imaging mode or with conventional long exposures, tend to smooth the atmosphere-induced speckles to the point where fixed speckles become apparent. These originate in the fixed bumpiness of the main mirror and the smaller optics, including the adaptive mirror. The fixed amplitude pattern in the pupil, caused by secondary obscuration, spiders, mirror dirt, etc. also contributes, but to a lesser degree since the Lyot stop is configured to remove much of the corresponding straylight. Attaining a more accurate measurement of static residual bumpiness in the ShackHartmann wave sensor should improve very much the situation. The accuracy of such measurements being rather immune to the photon noise affecting fast wave corrugations, there is hope of significant improvements. An easier but less efficient method, which can be used simultaneously, involves adding bias signals, randomly variable in space and time, to the actuators. The issue will be analyzed in more detail in a forthcoming article.

\section{Conclusion}

We have shown the feasability of imaging binary systems with the dark-speckle coronagraph, up to difference magnitudes of 4 and despite acquisition problems. Longer integrations will be required to achieve the maximum ratio 
predicted by the dark-speckle model (see Sect. 3.1). Our results on $\delta$ Per and $\eta$ Psc were affected by the presence of static optical defects mostly due to the pupil stop and to the AO system, which limits the speckle smoothing. The fixed residual speckles remain the dominant source of noise in these coronagraphic data and should be removed from future systems. If the phase distribution in the detected speckled coronagraphic image could be recorded, the fixed contribution could in principle be attenuated by adaptive optics, as shown by Malbet et al. (1995).

Our preliminary results cannot yet indicate whether extra-solar planets will be actually observable with ground-based telescopes, as suggested by the theoritical analysis (Boccaletti et al. 1998). The situation is much more favourable in space, where the dark speckles can be "boiled" leisurely, using one or ten second cycles of actuator switching and exposing. Following $8 \mathrm{~m}$ orbital telescopes, large interferometric arrays of free-flying telescopes will also be usable for coronographic dark speckle imaging (Labeyrie 1998).

Acknowledgements. We are grateful to the Office National d'Études et de Recherches Aérospatiales for providing their adaptive optics system. We thank Jean Gay, Yves Rabbia and Pierre Baudoz for a convivial sharing of the telescope light, and to the staff at the Observatoire de Haute-Provence for their efficient support.

\section{Appendix}

A formalism is exposed in Wynne (1979), but an easier derivation is possible. We have the following wavelength dependent relationships (according to the notation of Fig. 2):

Basically, the diameter of the Airy disk is:

$y^{\prime}(\lambda)=\frac{1.22 \lambda}{2 u^{\prime}(\lambda)}$.

The Lagrange invariant is:

$y . u=y^{\prime}(\lambda) \cdot u^{\prime}(\lambda)$.

The Wynne correctors make the exit pupil chromatic and allow the approximation: $u^{\prime}(\lambda)=a \lambda$. $a$ is a constant determined at $\lambda_{0}$ where the correctors do not have any effect:

$a=\frac{u^{\prime}}{\lambda_{0}}=u \frac{y}{y^{\prime}} \frac{1}{\lambda_{0}}=\frac{u}{\gamma} \frac{1}{\lambda_{0}}$

$\gamma$ is the magnification induced by the achromatic doublets:

$\gamma=\frac{y^{\prime}}{y}=\frac{f_{2}}{f_{1}}$

hence:

$u^{\prime}(\lambda)=\frac{u}{\gamma} \frac{\lambda}{\lambda_{0}}$

and:

$y^{\prime}(\lambda)=1.22 \frac{\lambda_{0} \gamma}{2 u}$

The Airy disk diameter no longer depends on wavelength.

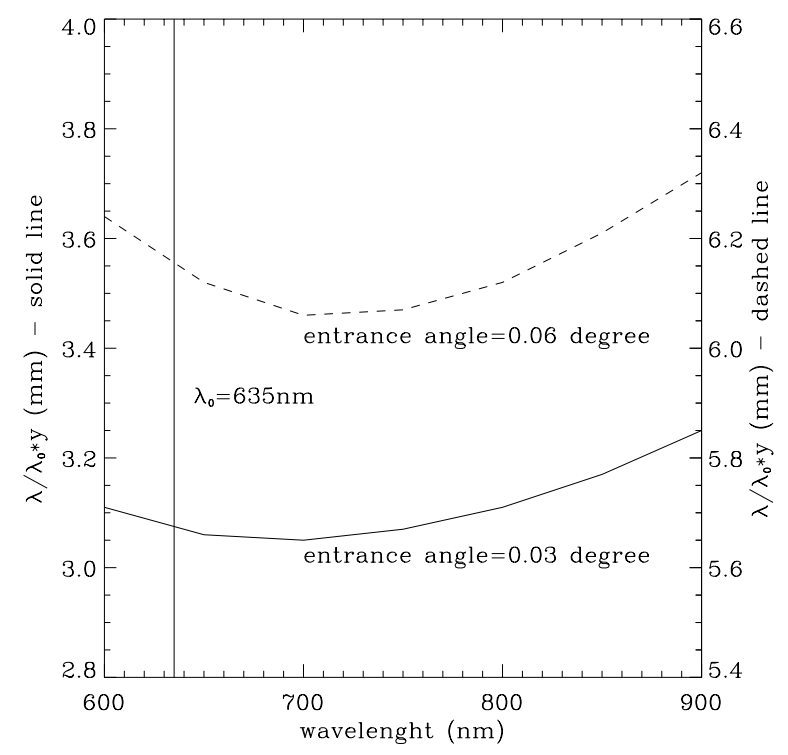

Fig. 6. Final radial chromatism on the detector $(f / 976)$ calculated from a numerical simulation, after correction by the Wynne triplets, for 2 different locations in the field (solid line $\theta=0.03^{\circ}$, dashed line $\theta=0.06^{\circ}$ )

\section{References}

Angel J.R.P., 1994, Nat 368, 203

Bacon R., Adam G., Baranne A., et al., 1995, A\&AS 113, 347

Boccaletti A., 1998 (to appear in IX ${ }^{\mathrm{e}}$ Rencontres de Blois)

Boccaletti A., Ragazzoni R., Labeyrie A., 1998 (to appear in A\&A)

Bonneau D., Josse M., Labeyrie A., 1975, proc. Utrecht Symp. de Jager/Nieuwenhuijzen eds. Reidel, p. 403

Conan J-M., Mugnier L., Fusco T., 1998 (to appear in Applied Optics)

ESA, 1997, The Hipparcos Catalogue, ESA SP-1200

Gay J., Rabbia Y., 1996, CR. Acad. Sci. Paris, t. 332, Serie II b, p. 265-271

Gezari D.Y., Crotts A, Danchi W., et al., 1997, Columbia University and Ball Aerospace Systems Division, proposal in response to NASA AO-96-OSS-3

Gezari D.Y., Crotts A, Danchi W., et al., 1997, Columbia University and Ball Aerospace Systems Division, proposal in response to NRA 98-GSFC-1

Gray D., 1997, Nat 385, 795

Gray D., 1998, Nat 391, 153

Labeyrie A., 1995, A\&A 298, 544-548

Labeyrie A., 1998 (in preparation)

Lyot B., 1930, Comptes rendus 191, 834

Malbet F., Yu J.W., Shao M., 1995, PASP 107, 386

Malbet F., 1996, A\&AS 115, 161-174

Mayor M., Queloz D., 1995, Nat 378, 355

Peacock T., Verhoeve P., Rando N., et al., 1998, A\&AS 127, 497

Roddier F., Roddier C., 1997, PASP 109, 815

Thiébaut E., 1994, A\&A 284, 340

Vakili F., 1990, PhD Thesis, Nice University

Wynne C.G., 1979, Opt. Comm. 28, 21 